\title{
Development of a Cloud Computing Interoperability-Based Service Certification
}

\author{
Kangchan Lee, Chulwoo Park and Hee-Dong Yang** ${ }^{1}$ \\ ETRI/UST \\ Hanyang University \\ Ewha Womans University \\ chan@etri.re.kr,chojema@gmail.com,hdyang@ewha.ac.kr
}

\begin{abstract}
Cloud computing and related services, in combination with mobile computing, are becoming a major building block of new IT convergence. Therefore, cloud interoperability based service evaluation certification demands precise and professional requirements. This study presents the quality, interoperability, and security as three key areas of overall cloud services. It also presents a proposal to evaluate the quality and interoperability through an existing cloud service certification system considering practical convenience and reality in establishing the certification system.
\end{abstract}

Keywords: Cloud computing, Interoperability, Cloud Service

\section{Introduction}

Since 2000, almost ten years after its emergence, cloud computing together with mobile computing has been a major building block of the development process of information technology infrastructure.

Gartner's annual top ten technology trends have continuously identified clouding computing.

Clouding computing first appeared in the list in 2009 and remained until 2012; since 2013, clouding computing has been separated into several detailed areas.

- $\quad$ Personal Cloud (2013) $\rightarrow$ The Era of Personal Cloud (2014)

- Hybrid IT and Cloud Computing (2013) $\rightarrow$ Hybrid Cloud and IT as Service Broker (2014)

- Cloud/Client Architecture $(2014,2015)$

This technology trend reflects that cloud computing is evolving into specific and detailed areas. Therefore, it is extremely important to establish a technical, procedural, and institutional plan to support its diversity and extensibility.

There have been various efforts to develop standards, security, quality, and interoperability. This study focuses on interoperability and addresses development of a certification system that can be operated after the concept of interoperability is institutionally rooted. This study summarizes views and previous studies on interoperability, examines similar certification systems that are applicable nationally and internationally, and derives a certification system reflecting various requirements and functions that can ensure interoperability of cloud services.

* Corresponding author 


\section{The Concept of Cloud Computing Interoperability}

Interoperability is the ability for systems and products to work well with other systems and products without any special efforts from the customer. Its importance in determining the quality of information technology projects has been rising steadily, and its use has been extended to many areas.

There has been a widely held recognition that security is the largest obstacle preventing the spread of cloud computing because of potential data loss due to neglected customer data management or poor management by the service provider. Consequently, many corporate users tended to build in-house database systems and use only the cloud service provider's public applications.

Although an open network is needed for clouds, the current network is designed to be a closed network, which reduces interoperability. Thus, many companies are undergoing various difficulties in unifying the IT system with clouds and realizing increased productivity and cost savings. Cloud service providers must design an improved interoperability platform and establish industry standards for data mobility. Moreover, they can attract more corporate customers by providing improved enterprise tools that raise the quality of internal data application rights and security assurance.

Terms related to interoperability include intercloud, open cloud computing interface, and cloud data management interface.

Currently, there are many definitions of intercloud. Intercloud [10] is a "cloud of clouds" that are globally connected [8]. Furthermore, it is a concept extended from the Internet, that is, the "network of networks". The term intercloud was first used in the cloud computing environment in 2007. Since then, intercloud has become popular and is used to describe the data center of the future.

Intercloud computing enables on-demand allocation within the range of movement of transactions through the interaction of computing, storage, network, and cloud resources and cloud systems [4].

The Open Cloud Computing Interface consists of open communities. Specifications for how infrastructure service providers provide their computer, data, and network resources through a standardized interface are delivered by the Open Grid Forum.

The Cloud Data Management Interface (CDMI), a SNIA standard, specifies protocols for self-provisioning as well as accessing and managing cloud storage. CDMI evaluates the cloud storage system's capability, evaluates and allocates containers and objects, manages users and groups, carries out evaluation control, accesses meta data, makes arbitrary queries, uses repeated queues, specifies interval memory and holds for compliance, uses a logging system, makes payments, moves data among cloud systems, and defines secure HTTP management to export data through other protocols such as iSCSI and NFS.

\section{Industry Trends and Previous Studies}

\subsection{Cloud Industry Trends}

According to the 2013 Korea Media Panel Survey, recent national cloud service usage can be summarized as follows [5].

- In 2013, cloud service was used only by $7.6 \%$ based on a very narrow usage trend by younger users including those in their 20s and specialty occupation professionals.

- The biggest obstacle to the wide-spread use of cloud service can be found in terms of the ability to use the Internet.

- Although the ability to perform basic file upload/download operations is necessary to be able to use cloud services, only $58.6 \%$ of respondents said that they can open email-attachment files. 
- It is estimated that a combination of various causes, including the personal data privacy issue, large file-based media and work usage status, and lack of recognition of the need for data backup, contributed to the low national cloud service usage rate of $7 \%$.

- The most competitive companies in the national cloud market include Naver and other portals, whereas three major communication companies and Western companies such as Apple and Google have had relatively weak influence.

- There is a need to pay attention to the recent emergence of Baidu and other Chinese companies, as they are known to offer a type of media file streaming service as well as offering free storage in terabytes that are much larger than what the previous companies offer.

- Advanced cloud services that combine various software, rather than simply providing data backup, are beginning to unfold.

- Since the potential for future growth is paradoxically huge due to the currently low rate of cloud service utilization, it is necessary to establish diverse measures to promote the national cloud service market and prepare for the influx of the Chinese companies.

The US is stimulating the adoption and use of clouds in the public procurement areas by carrying out FedRAMP since 2012, and Japan is promoting the use of clouds to enhance the data backup and business continuity after the Great East Japan Earthquake.

\subsection{Related Research Trends}

In recent years, the demand for policy, theory, and practical guidelines centered on and related to cloud computing has been high, and many studies have been carried out for standardization; however, it is very rare for standardization bodies to define related problems and requirements and to present specific deliverables. More effort is needed when dealing with details such as interoperability, and Jeong [1] argues that specific standardization items and reference models are necessary to improve semantic interoperability.

Presently, there is almost no cloud computing standardization from technical standpoints, and the standardization items currently offered only deal with conceptual definitions and reference structures in related areas rather than technical aspects.

The ISO/IEC JTC 1/SC 38 is a committee on Distributed Application Platforms and Services (DAPS) [11]. Initially, it consisted of two Working Groups for standardization of web services and Service-Oriented Architecture (SOA). However, with more social attention on cloud computing and various research studies, the Study Group on Cloud Computing (SGCC) verified the standardization validity and theme, and its activities resulted in forming a new working group, WG3: Cloud Computing, for cloud computing. SGCC submitted a final report in 2011 [6], and defined standardization issues related to functional interface, management interface, portability, and security.

In the ITU-T SG 13 cloud computing meeting held in Geneva, Switzerland in February 2014, the following topics were discussed.

- Desktop virtualization standard approval

- Cloud computing based big data

- NaaS standards development

- IaaS standards proposal development

- End-to-end cloud computing lifecycle management

The Open Cloud Consortium (OCC) is a non-profit consortium led by academia and industry whose members include Cisco, Yahoo, Johns Hopkins University and NASA [7]. OCC favors an open cloud computing environment. In particular, the Open Science Data 
Cloud (OSDC) Working Group focuses on big data cloud management and operation for scientific data.

TTA PG420 is a national standardization organization, and this project group has actively performed cloud computing standardization activities. TTA PG420's standardization activities focus on service framework, requirements, use case, and terms, and development of specific technical standards is needed.

\section{Interoperability based Cloud Service Assessment Elements and Certification Indicators}

\subsection{Need for Interoperability Certification}

The "bill for cloud computing development and user projection" that is being actively discussed in the Parliament mainly deals with development and use of cloud computing services such as support for small businesses and priority consideration for introduction to national agencies.

Although there is no mention of "certification" of related services, it does not guarantee pure autonomy by employing terms such as recommendations, responsibilities, and penalties.

Article 16 in Chapter 3 specifically mentions ensuring interoperability.

"Article 16 (Ensuring Interoperability) The Minister of Science, ICT and Future Planning can recommend that cloud computing service providers establish a cooperative system to ensure interoperability of cloud computing services if needed."

Failure to follow this recommendation does not result in a penalty, but the service provider must pay attention to this information if the bill passes as is, and the certification system in the related fields must be properly ensured to confirm procedural and institutional implementation of the recommendation.

\subsection{Interoperability-Based Cloud Service Assessment Elements}

Ensuring and achieving interoperability of cloud services requires an incremental approach used in similar areas. In order to achieve the overall goal, it is important to establish problems to be solved in the short-, medium-, and long-term and their priorities, and to identify the degree of achievement.

With the current and strong individual, organizational and governmental interests as well as the proliferation of the mobile environment, the use of cloud computing and services is predicted to bring significant changes in life, business, administration and all other sectors of society. However, any interoperability problem in the process to unify information systems to clouds, especially at the industrial organizational level, will become a barrier to the introduction and operation of relevant systems.

Only after this problem is resolved, the motivation to move information related services and systems to cloud will grow, which will lead to the reduction in associated costs and increase in productivity and efficiency. In addition, cloud computing will be able to solve the issue of many companies' keeping systems in their network that are not directly related to core competencies.

It is desirable to take the sociotechnical approach taken by other areas in order to understand cloud computing from the interoperability viewpoint. With the ultimate goal of confirmation after applying this approach, viewpoints are first divided into technical and social.

From the perspective of technical issues and requirements, there is an emphasis on interlocking technology at the level including API and middleware for interoperability that recognizes the uniqueness of each service. 
Obviously, standardization that is oriented to promote industrial activities and to increase information security and user protection and satisfaction, not to regulate the service industry, should be supported. An atmosphere that allows the service providers to participate in the standardization process with the expectation of increased industrial activities and to derive valuable results should be established, and ultimately market- and technology-driven industrial activities should be pursued.

This study first categorizes the capability of cloud service interoperability in the context of social approach that includes procedures and policies, rather than in the context of technical approach to ensure interoperability, into four categories: scalability, universality, continuity, and specialty.

Scalability refers to changing the system size to guarantee seamless interoperability, and includes five functions that are policy establishment, quality management, platform management, interface management, and internal management.

Universality includes the capability for prevention and quality measurement and service instruction operation in addition to policy establishment, quality management and internal management that overlap scalability.

Next, continuity has the property of offering uninterrupted service when transferring to a different provider's service or switching to a different type of service when cases, such as the service provider change, make continuous use of existing service is difficult. Continuity includes the service's lasting capacity, variety of service types, change management, service provider independence, and guarantee of the service user's rights.

Finally, specialty is also an important capability because the ultimate goal of interoperability is to "use the other like the same" although specialty seems to exclude interoperability at first glance. Specialty includes service quality assurance, interface planning, change management, internal management, and service quality management.

An "interoperable cloud service" absolutely requires all of these four capabilities.

\section{Cloud Computing Interoperability Certification System}

\subsection{Cloud Service Certification Related Procedure}

5.1.1. National Cloud Service (Quality) Certification Procedure: The Korea Communications Commission said that it would award the first national private cloud service certification to KT's enterprise cloud service, nCloud biz, on June 18, 2012. The cloud service certification procedure is part of the "cloud service expansion and competitiveness strategy" announced by inter-agency collaboration in May 2011. The procedure evaluates the quality of various areas, such as cloud service quality and information security as well as service continuity and grants certification by the Korea Communications Commission if certain system and process are secured. Since the national cloud service certification procedure began in 2012, only two certifications to KT and SK Telecom have been awarded until December 2013, and there has not been a more recent application.

Cloud service certification is a certification granted to services that passed existing certification evaluation criteria, and more demanding conditions can ensure the quality of the service. However, these conditions can be an obstacle to expanding the certification procedure. For example, the cloud service excellence SLA certification can only be granted to the companies who earned general certification and satisfy three requirements that include more than $99.5 \%$ service availability, Information Security Management System (ISMS) certification, and security related certification such as ISO 27001. When there requirements are met, there is no need to obtain separate certification and there is a limited number of target companies and services that can meet these requirements.

The Korea Association of Cloud Service, a practical organization in charge of related work, was renamed to the Korea Association of Cloud of Industry after the approval from 
the Ministry of Science, ICT and Future Planning on September 11, 2014. Although part of its services includes cloud service certification operation, the cloud service certification and excellence SLA cloud service certification homepage that officially manages related information does not reflect the association's name change, and does not contain enough related information.

5.1.2. Korean GS (Good Software) Certification: GS certification, operated by the National IT Industry Promotion Agency, is a certification procedure related to the software quality. It is a nationally granted certification following the Software Industry Promotion Act and The Software Industry Promotion Act Decree. Certification is granted after testing the software functionality, reliability, efficiency, usability, maintainability, portability, performance, interoperability, and conformity based on the ISO international standards. Many companies including small businesses are participating since it is highly relevant to the cloud computing area centered around SaaS and widely usable.

The certification helps to secure the reliability authorized by a certification agency designated by the Ministry of Knowledge Economy, and is used for the certified company's national and international marketing and promotion. The certified software product can be looked up on the relevant web page to reference the product name, company name, and certification number, which can also be downloaded as an Excel file.

5.1.3. Korea's Service Quality Evaluation System: The Ministry of Science, ICT and Future Planning has been piloting the "cloud service quality evaluation system" [9] since January, 2014. This service was developed by the Korea Internet \& Security Agency and the Korea Cloud Service Association to induce voluntary quality improvement from national and international cloud service providers and to provide quality information so that the user can choose an appropriate service.

The system provides quality information on seven services that include five national services - Olleh ucloud biz (KT), Tcloud biz (SKT), LGU+, Hostway, and Innogrid - and two international services-Amazon AWS and Rackspace.

This evaluation system plans to improve and complement the certification procedure by linking to the 'cloud service certification system' that evaluates on paper only and whose effectiveness is being pointed out so that it can be used for safety checks needed on the use of private clouds by the public sector. The significance of this evaluation system is in effecting a foundation for cloud industry expansion through improving the cloud service user's confidence.

What is needed includes absolute as well as relative comparison among services, longitudinal and long-term rather than cross-sectional and intermittent information, and information on exception management status that contains anomaly and actions. Moreover, specific content needs to be confirmed by details provided in the drill-down type information. Although the quality status of various services is shown in a weather forecast form, the level of information utilization can become relatively lower due to the large amount of information provided by the weather information in considerable breadth and depth. In addition to the past- and present-oriented information, predictions based on the current information are also needed. Traffic information systems show future traffic information in addition to the current traffic information.

5.1.4. The US Cloud Computing Security Certification System: The US General Services Administration (GSA) announced the operation of FedRAMP, a certification program for public sector cloud security [3]. It can be understood as a specific certification on security rather than a certification on the entire cloud services.

FedRAMP is a standardized government program for security assessment, certification, and continuous monitoring of cloud products and services. By unifying the security 
evaluation and certification that used to be done by different agencies, it brings cost, time, and labor savings. Cloud services that received FedRAMP security certification can be unilaterally introduced to other agencies without any additional certification process. The US federal agencies are required to only use enterprise services that received FISMA certification. Therefore, any introduction of cloud services to federal agencies must be given the Authority to Operate (ATO) in accordance with FISMA and must use FedRAMP. This reuses temporary certification and security evaluation packages in FedRAMP secure storage. FedRAMP provides temporary certification and security evaluation packages only. The actual responsibility to examine security evaluation packages, evaluate their effects, and grant certification belongs to a federal agency. Similar national certifications include ISMS certification and CC certification. It is difficult to say that these certifications reflect a cloud service environment.

5.1.5. Open Certification Framework (OCF): OCF is a cloud security control framework established by the non-profit Cloud Security Alliance (CSA). CSA OSF is built based on three stages of trust, and each stage offers incremental levels of visibility and transparency on the cloud service provider's operation and provides the cloud consumer with higher levels of quality [2].

\subsection{Proposed Interoperability Certification System}

5.2.1. Certification Process: A look at the cloud service certification operation shows that the Korea Association of Cloud Industry, which is the agency that carries out the certification, examines documents submitted by the applicant organization, makes a site visit as needed for further investigation, and makes a comprehensive evaluation. Evaluation comprises four steps: preparation, examination, certification, and follow-up. Certification is valid for two years from the date of issue, and can be revoked after reevaluation. However, unlike ISMS, PIMS certification examination is commissioned to and operated by the Korea Online Privacy Association through a consignment agreement. The examination operation includes certification consultation and application acceptance, contract agreement, receipt and payment of certification fees, examiner liaison and examination committee formation, certification examination, examination result report, and certification committee review requests. It is similar to the cloud service certification done by the Korea Association of Cloud Industry responsible for the Korea Communications Commission.

5.2.2. Proposed Certification System Operation: Private evaluation agency use: This study first proposes a certification system that utilizes accredited private evaluation agencies. The current KCSC procedure consists of requests that utilize the talent pool of the Korea Association of Cloud Industry. The disadvantage of the existing talent pool configuration and evaluation system is that they lack some objectivity because of their probability of being arbitrary. To overcome this disadvantage, FedRAMP operates by having a designated professional evaluation agency, 3PAO. This dependence on a designated agency that offers credibility and trust can be proposed for domestic use.

Although there may be a primary problem of determining the degree of credibility, it could work after a discussion of the eligibility requirements. The relevant certification authority can monitor the overall certification process and be responsible for maintenance and update processes. The certification agency is to be open internationally, not limited to a domestic agency, so that the proof of certification results may be regarded as part of the certification process.

Unified use of cloud service certification system and interoperability evaluation elements: The current cloud service certification system's seven assessment items and their detailed criteria do not include interoperability. Interoperability is added to the 
existing seven assessment items as the eighth item, and the detailed criteria can be designed to extract and integrate elements included in interoperability. This method can overcome the disadvantage of operating many certification systems related to cloud services vertically. Moreover, it has the advantage of eliminating the inconvenience of having to grant and receive certifications on separate items such as interoperability and security for relevant agencies and service providers, and seeking the integration and management of a macro certification system.

On one hand, in order to minimize the number of evaluation items for compact evaluation and operation, evaluation items may be reorganized and evaluation items may be readjusted. Considering the content of interoperability evaluation items, it is desirable to include scalability and service continuity, which are existing cloud service certification items, in interoperability.

However, as examined above, although the cloud development legislation is a recommendation, it provides a basis for securing interoperability. Therefore, it is better to ensure openness, objectivity, and continuity by dividing the cloud computing service evaluation field into three major subfields-interoperability, quality, and security, establishing certification and maintenance systems for each subfield, and operating an integrated online evaluation and referral system, than to discuss its status as a part of the overall service evaluation item. For the quality and interoperability, it is more practically compelling to integrate them with the existing cloud service and manage them, when compared with other operating methods, for the effectiveness of the certification system and convenience of the service providers within the industry.

\section{Conclusion and Policy Proposal}

Cloud computing and related services, combined with mobile computing, have become a major component of new IT convergence. They are also important service items of main global IT companies such as Apple, Google, Amazon, Facebook, and Microsoft in addition to taking their place as a significant factor responsible for day-to-day activities and businesses of individuals, companies, and governments.

After this quantitative spread to an extent due to related services' rapid development and deployment, institutional support to ensure service continuity as well as quality evaluation is presently needed. In this context, there is a demand for evaluation and certification of technology and services, and a national cloud service certification procedure is in effect. Although the evaluation items included in the procedure were initially precisely designed and planned, they lack the criterion for 'interoperability'. Rectifying this lacking can reflect the necessity for interoperability presented in this study.

Evaluation through the certification system with the indirect means of making the service provider secure cloud interoperability is of course needed. However, at the national level, it can be a weapon to prevent losing the initiative in securing stable information technology and in relationships among globally competitive information industries.

Therefore, cloud interoperability based service certification demands precise and professional elements. This study presents the quality, interoperability, and security as three key areas from the overall cloud service viewpoint. This study also presents a proposal to evaluate the quality and interoperability through existing cloud service certification systems after considering practical convenience and reality to establish a certification system.

The proposal includes the properties related to interoperability in the existing cloud service evaluation item and provides a certification procedure that comprises the service provider's application and the relevant agency's evaluation process. When the certification is complete, the certification documents indicates the effective period. Relevant evaluation teams must follow the certification committee member eligibility 
used in existing cloud service evaluation, which is managed by the Korea Association of Cloud Industry that has been in charge of cloud service certification.

The future will bring unimaginably diverse business models that belong to the cloud industry including cloud computing and cloud computing services. Moreover, it may be difficult to define boundaries among composite services combined with different forms of computing. This makes it difficult to predict the property changes due to the evolution of clouds.

Although frequent changes to evaluation items and criteria are problematic to ensure the certification operation's objectivity and continuity, steady upgrades and evolution of certification systems should parallel the speed of technology development and reality. Since efforts by the Ministry or related agencies may be limited, an alternative may be to cultivate a third authorized certification agency and secure a management system for this agency.

\section{Acknowledgements}

This work was supported by Institute for Information \& communications Technology Promotion(IITP) grant funded by the Korea government(MSIP) (R0166-15-1033, Development of Cloud Computing Standards) and by the MSIP(Ministry of Science, ICT\&Future Planning), Korea, under the ITRC(Information Technology Research Center) support program (NIPA-2013- H0301-13-4006) supervised by the NIPA(National IT Industry Promotion Agency).

\section{References}

[1] D. Jeong. A Standard Reference Model for Semantic Interoperability in Cloud Computing, Journal of The Korea Society of Computer and Information. 17, 8 (2012), pp. 71-80.

[2] J. Y. Choi, E. J. Choi and M. J. Kim. A Comparison Study between Cloud Service Assessment Programs and ISO/IEC 27001:2013, Journal of Digital Convergence. 12, 1 (2014), pp. 405-414.

[3] K. S. Kou. A Study on Security-Enhanced Cloud Service E\&C (Evaluation and Certification Scheme, Journal of Security Engineering. 9, 6 (2012), pp. 481-494.

[4] FG Cloud Technical Report Part 1. February (2012)

[5] ] KISDI STAT Report, (2014) January 25, pp. 7-11.

[6] ISO/IEC JTC 1/SC 38/SGC, "Final SGCC Report: Study Group Report on Cloud Computing," SC38N430, (2011)

[7] Open Cloud Consortium, http:/opencloud-consortium.org

[8] http://samj.net/2009/06/intercloud-is-global-cloud-of-clouds.html

[9] http://www.cloud-qos.or.kr

[10] http://www2.computer.org/portal/web/csdl/doi/10.1109/ICIW.2009.55

[11] ISO/IEC JTC 1/SC 38 - Distributed Application Platforms and Services, http:/www.iso.org/iso/ jtc1_sc38_home

\section{Authors}

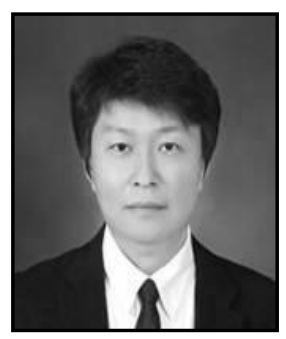

Kangchan Lee, he has been working for ETRI since 2001. He started in Protocol Engineering Center to develop the technology and standards for Next Generation Web. Until now, he has been participated several standardization projects which are related to Web technologies, such as Ubiquitous Web Services, Mobile Web, etc, and his major research interests are Next Generation Web, Cloud Computing, Future Networks, distributed system integration, database integration technology, digital library, information retrieval and database, and structured document, etc. $\mathrm{He}$ has also been actively involved in international and domestic standardization activities. Since 2005, he is working with ITU-T to develop the Web-based convergence service standard in NGN 


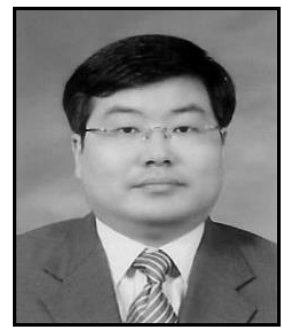

environment with several editorships in Study Group 13 of ITU$\mathrm{T}$. Also he is now the Rapporteur of Q17 (Cloud Computing requirements) at ITU-T SG13 since 2012. He is also a Professor in department of Information and Communication Network Technology at University of Science and Technology in Korea. Information and Communication Network Technology

Chulwoo Park, he is an Adjunct Professor at Hanyang University. He has a Ph.D. and a master's degree from Seoul National University in Management Information Systems. He previously was a Research Associate in the Institute of Management Research at SNU. He had worked for an IS department in a manufacturing company and was a Brain Korea Assistant Professor in SNU. He teaches researches and publishes in the fields such as IS management, information strategy, ecommerce, business computing and Internet business models.

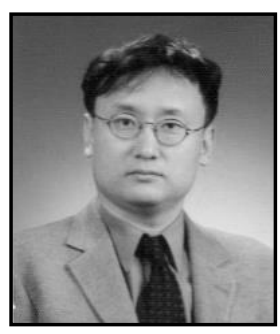

Hee-Dong Yang, he is a Full Professor in Ewha School of Business at Ewha Womans University in Korea. He has a Ph.D. from Case Western Reserve University in Management of Information Systems, and earned bachelor's and master's degree from Seoul National University (School of Management). He previously was an Assistant Professor at the University of Massachusetts-Boston. His research interests include technology standardization policy, management of innovative technology, B2B transactions, (smart) mobile business, adoption of information technology, organizational impact of information technology, team mental model, and strategic use of information systems. His papers have appeared in ISR, JAIS, I\&M, DSS, JSIS, EJIS, IJHCS, IJEC, JITM, BJM and HR, and also have been presented at many leading international conferences (ICIS, AMCIS, HICSS, Academy of Management, ASAC). 